\title{
Clinical Usefulness of Cardio-ankle Vascular Index, Local Artery Carotid Stiffness and Global Longitudinal Strain in Subjects with Cardiovascular Risk Factors
}

\author{
Vitantonio Di Bello, lacopo Fabiani, Enrico Calogero, Paolo Colonna' 1 , Scipione Carerj², Francesco Antonini Canterin ${ }^{3}$, Frank Benedetto ${ }^{4}$, \\ Salvatore La Carrubba ${ }^{5}$, Nicola R. Pugliese, Valentina Barletta, Lorenzo Conte
}

Department of Cardiothoracic, Cisanello Hospital, University of Pisa, Pisa, 'Division of Cardiology, Bari Hospital, Bari, ${ }^{2} \mathrm{G}$. Martino Hospital, Division of Cardiology, University of Messina, Messina, ${ }^{3}$ Santa Maria Degli Angeli Hospital, Division of Cardiology, Pordenone, ${ }^{4}$ Division of Cardiology, Bianchi-Melacrino-Morelli Hospital, Reggio Calabria, ${ }^{5}$ Villa Sofia-Cervello Hospital, Division of General Internal Medicine, Palermo, Italy

\section{INTRODUCTION}

The role of the italian society of cardiovascular echography in the preventive cardiology

Atherosclerotic cardiovascular disease (CVD) is a chronic disorder developing insidiously throughout life and often asymptomatic for a long period. It is the major cause of premature death in Europe and of mass disability.

The World Health Organization has stated that over three-quarters of all CVD mortality may be prevented with adequate changes in lifestyle.

The success of preventive measures depends on the accurate identification and treatment of individuals who are at risk for cardiovascular (CV) events (risk prediction).

Risk prediction, till now, relies mainly on the assessment of risk factors, but traditional ones (including age, sex, smoking habits, and lipid profile) do not identify everyone who will eventually develop CVD.

The net result is that as many as half of individuals who develop coronary heart or cerebrovascular disease have only one if none of traditional risk factors and the most disappointing element is that for such individuals prevention strategies are not undertaken. However if we consider those with few or none clinical risk factors all together, they represent the group of subjects with the largest number of cardiovascular events. ${ }^{[1,2]}$

This consideration prompts the emergence of potential novel screening tests.

Actual imaging biomarkers detect the presence of subclinical CVD but, with few and recent exceptions, are of limited value

\begin{tabular}{|l|l|}
\hline \multicolumn{3}{|c|}{ Access this article online } \\
\hline Quick Response Code: & Website: \\
& www.jcecho.org \\
& \\
\hline
\end{tabular}

for characterizing earlier stages, when subclinical disease is not always apparent.

Global risk scores, although designed to estimate risk across a continuous range from $0 \%$ to $100 \%$, have most commonly been advocated as a method by which patients can be categorized in broad terms as "low risk," "intermediate risk," and "high risk." In general, patients are deemed to be high risk if they are found to have a global risk estimate for hard coronary heart disease events of at least $20 \%$ over 10 years. The threshold between low and intermediate risk is not uniform, since some propose a lower cutoff value of $6 \%$ risk over 10 years, whereas others use a value of $10 \%$ over 10 years..$^{[1,3]}$

European CV risk charts as well as national ones (i.e., Progetto Cuore in Italy) usually account for common and well-known risk factors, as well as age, sex, smoking habits, cholesterol, and blood pressure levels. Anyway, it is dramatically clear that many $\mathrm{CV}$ events happen in otherwise healthy subjects, with no exposure to any of the previously cited risk factors. This claims for a deeper approach for stratification.

\section{The Role of the Italian Society of Cardiovascular Echography in the Preventive Cardiology}

The identification of asymptomatic patients at high CV risk is a great challenge. Ultrasound, the most commonly used

Address for correspondence: Dr. Enrico Calogero, Dipartimento Cardio-Toraco-Vascolare, Ospedale Cisanello, Via Paradisa, 2 - 56100 Pisa (PI), Italy. E-mail: enrico.calogero@gmail.com

This is an open access article distributed under the terms of the Creative Commons Attribution-NonCommercial-ShareAlike 3.0 License, which allows others to remix, tweak, and build upon the work non-commercially, as long as the author is credited and the new creations are licensed under the identical terms.

For reprints contact: reprints@medknow.com

How to cite this article: Di Bello V, Fabiani I, Calogero E, Colonna P, Carerj S, Canterin FA, et al. Clinical usefulness of cardio-ankle vascular index, local artery carotid stiffness and global longitudinal strain in subjects with cardiovascular risk factors. J Cardiovasc Echography 2017;27:81-7. 
noninvasive tool for the evaluation of $\mathrm{CV}$ anatomy and function, plays an important role in prognostic evaluation. Worldwide, approximately 17 million people are living with the consequences of atherosclerotic CVD; every year, millions die from its consequences. Curative care is not able to cope with the magnitude of the problem, and new solutions must be sought to combat the global epidemic of heart attacks and strokes. We know what to do, but we do not know how to implement. To meet this challenge, our association faces important responsibilities. Preventive cardiology deserves a central stage in the Italian Society of Cardiovascular Echography (SIEC), and we will assure that this position is being taken. The SIEC is synonymous with the excellence in preventive $\mathrm{CV}$ care and research. ${ }^{[4]}$ For all these reasons, efforts are increasingly being targeted toward early diagnosis for intervening at an earlier stage of disease. Italian Society of Echocardiography has been involved from its origins in preventive field, developing several research programs, ${ }^{[4-14]}$ including Disfunzione Asintomatica VEntricolare Sinistra ${ }^{[15]}$ study (on cardiac field), and considering deeply prognostic implications of vascular disease.

\section{Electrocardiogram}

The routine or screening electrocardiogram (ECG) is a common office procedure, even in the current era of cost-conscious health care. There is a hard debate if the resting ECG signs, both major or minor, are able to discriminate between healthy asymptomatic individuals and those likely to develop cardiocirculatory events. Recent studies have demonstrated that in older patients, this simple technique (ECG) could be useful to discriminate individuals with an increased risk of $\mathrm{CV}$ events. Many ECG alterations, in heart failure population, have strong prognostic and therapeutic implications. Due to its relatively low cost, easiness of use, and widespread availability, ECG remains an important instrument for large-scale preventing interventions.

\section{Noninvasive Vascular Imaging: Conventional Indices and Stiffness Evaluation}

Over the last few years, noninvasive imaging of atherosclerosis and CV mechanics has increasingly been used in clinical practice. It is appalling to realize, however, that there is a lack of data regarding the impact of such screening. Available evidence regarding atherosclerosis screening is limited, with mixed results regarding $\mathrm{CV}$ factors control.

\section{Carotid vessels}

A screening program that uses carotid duplex ultrasound (B-mode and Doppler) aims to detect individuals with asymptomatic carotid artery stenosis for several purposes.

1. To identify individuals at risk for $\mathrm{CV}$ event, particularly in the cerebrovascular and coronary circulations

2. To select individuals who need significant risk factor modification
3. To potentially intervene with carotid endarterectomy or carotid stenting to prevent a stroke.

Screening studies using carotid duplex ultrasound have shown that $4 \%-8 \%$ of individuals aged 50 years will have an asymptomatic $50 \%$ carotid stenosis.

Population-based studies have shown a correlation between the severity of atherosclerosis in one arterial territory and the involvement of other arteries. Therefore, early detection of arterial disease in apparently healthy individuals has focused on the peripheral arterial territory and on the carotid arteries. Risk assessment using carotid ultrasound focuses on the measurement of the intima-media thickness (IMT) and the presence of plaques and their characteristics. ${ }^{[7]}$

Cerebrovascular risk stratification is possible using a combination of clinical and ultrasonic plaque features. There is an independent association between carotid IMT and CV events ${ }^{\left[{ }^{[16]}\right.}$ However, there are few data showing that it improves measures of predictive performance. Many results underline the benefits of assessing these parameters only in limited cohorts of patient (i.e., older women).

Epidemiological studies have shown that, in the absence of detectable carotid stenosis, thickness differences of the intimal-medial layer in the common or internal carotid artery have independent prognostic significance for future $\mathrm{CV}$ events. Nevertheless, the predictive value is relatively small compared with the finding of a stenosis and the technique requires careful training and standardization. ${ }^{[7,8,14]}$

Although a variety of imaging modalities are available to visualize the abdominal aorta, in search of asymptomatic aneurysms, ultrasonography is the noninvasive procedure of choice for screening because of its widespread availability, reasonable accuracy, relatively low cost, and excellent safety. The sensitivity and specificity of ultrasound for the detection of abdominal aortic aneurysm disease are $95 \%$ and $100 \%$, respectively.

The assessment of arterial stiffness, a common feature of aging, exacerbated by many common disorders such as hypertension, diabetes mellitus, or renal diseases, has become an attractive tool for identifying structural and functional abnormalities of the arteries in the preclinical stages of atherosclerotic disease ${ }^{[5,13]}$ Arterial stiffness has been recognized as an important pathophysiological determinant of systolic blood pressure and pulse pressure (PP) increases and therefore the cause of $\mathrm{CV}$ complications, demonstrating also an independent predictive value for $\mathrm{CV}$ events. Although there are many techniques and indices currently available, their large clinical application is limited by a lack of standardization, with important difficulties to effectively measure, quantify, and compare. ${ }^{[17]}$ Moreover, information on "heart-vessel coupling disease," in which combined stiffness of both heart and arteries interacts to limit CV performance and its possible implications in different clinical conditions, is still not well known. 
Arterial stiffness can be assessed as local, regional, and systemic stiffness. Although systemic arterial stiffness may only be estimated, local and regional stiffness can also be measured. Direct measurement of arterial stiffness implies the measurements of change in arterial diameter and pressure at the same site ${ }^{[18]}$ This could be performed invasively or noninvasively. Diameter changes can be determined accurately, especially with high-definition echo-tracking systems, but the estimation of pressure changes at the same site may be difficult because of PP amplification and inaccuracy of all cuff sphygmomanometer systems. ${ }^{[19]}$ Indices of local stiffness are distensibility (the relative change in diameter with pressure), compliance (the absolute change in diameter or area with pressure), Peterson's elastic modulus (pressure change required for theoretic 100\% increase in diameter), and Young's elastic modulus (pressure change per square centimeter for theoretic $100 \%$ extension). A nondimensional index of local arterial stiffness frequently used is the stiffness index (ratio of logarithm [systolic/diastolic pressures] to relative change in diameter).

Pulse wave velocity (PWV) is accepted as the most simple, robust, and reproducible method to determine the regional arterial stiffness. ${ }^{[20,21]}$ There is a linear correlation between pulse speed along an arterial segment and arterial stiffness. To calculate PWV, the distance between the two sites at which the wave is being recorded is divided by the time of wave from the first to the second site:

$\mathrm{PWV}=$ distance/time

It is usually measured using the foot-to-foot velocity method from various waveforms at different sites (pressure, distension, or Doppler waveforms).

An important body of evidence links increased PWV, elevated $\mathrm{PP}$, and premature/increased arterial wave reflections with a higher risk of $\mathrm{CV}$ morbidity and mortality. ${ }^{[2]}$ Aortic stiffness has independent predictive value for all-cause and CV mortalities, fatal and nonfatal coronary events, and fatal stroke in hypertensive, diabetic, or renal patients, in elderly or healthy subjects and in the general population. ${ }^{[23,24]}$ The independent predictive value of aortic stiffness has also been demonstrated after adjustment to classical CV risk factors. Recent data from the Framingham Heart Study show that the prevalence of abnormal aortic stiffness increases steeply with advancing age in the community, especially in the presence of obesity and diabetes.

\section{Peripheral artery disease: Ankle-brachial index}

The ankle-brachial index (ABI) is the ratio of systolic blood pressure at the ankle and at the arm. It is measured with supine patient, usually with a sphygmomanometer and Doppler ultrasound probe. The precise technique and calculation of the ABI have not been standardized universally, but a common approach is to measure systolic pressure in both arms and at the posterior tibial and dorsalis pedis arteries in each ankle with the patient in a supine position. The ABI for each leg is then calculated as the higher pressure at the ankle divided by the higher of the left and right arm pressures.
In theory, the ABI might be used for two linked but different screening purposes.

1. To detect asymptomatic arterial disease in the legs to prevent progression to claudication or critical limb ischemia

2. To detect individuals at high risk of future $\mathrm{CV}$ events to initiate $\mathrm{CV}$ risk reduction measures.

Only $20 \%$ of major CV events occur in individuals with a history of CVD, while for those without such a history of risk factor scoring systems such as the Framingham risk score have limited accuracy. There is thus considerable interest in the potential of other markers to improve prediction of $\mathrm{CV}$ events. An ABI $<0.90$ has been associated consistently with a 2-4-fold increased relative risk of CV events and death. For the evaluation of screening, however, measures of validity, such as sensitivity, specificity, and positive and negative predictive values, are more relevant.

Recent evidence of B-mode evaluation of bilateral iliofemoral arteries provides an incremental yield in identifying subclinical atherosclerotic disease as compared to carotid evaluation alone. In particular, the Fuster-Narula Score, evaluating intima-media volume, is a promising tool for patients' stratification.

There is a large amount of evidence on the importance of arterial stiffness in predicting outcome in patients with CV risk factors. The accepted "gold standard" for measuring arterial stiffness nowadays is the carotid femoral PWV as reported by the recent guidelines on diagnosis and treatment of arterial hypertension.

Recently, other measures of arterial stiffness have been proposed and validated in different clinical scenarios, namely, the cardio-ankle vascular index (CAVI) and local carotid artery stiffness measured by a high-resolution ultrasound radiofrequency system to obtain the radiofrequency based quality arterial stiffness (RF-QAS) at common carotid artery level. The CAVI is a stiffness and arteriosclerosis indicator of thoracic, abdominal, common iliac, and distal arteries independent of arterial blood pressure. ${ }^{[25-28]}$ To obtain CAVI, the distance from the level of the aortic valve (i.e., the brachial level) to the measuring point (i.e., the ankle) is recorded, and then the time delay between the closing of the aortic valve to the variation detected in the arterial pressure wave at the set point is also recorded. ${ }^{[27,29]}$ Information for CAVI calculation, including PWV, blood pressure, and arterial pulse waveforms, can then be acquired through the ECG, cardiac phonogram, and pressure cuffs on the testing subject at the reference points. In comparison to PWV, CAVI has two distinctive aspects. First, CAVI changes in a short period that depends on alterations in circulatory condition. Second, CAVI reflects the state of contraction of smooth muscle rather than changes in blood pressure. ${ }^{[30]}$ Regarding the clinical and prognostic role of CAVI, there is an increasing amount of significant evidence, particularly from Eastern Asian countries, while in Europe and United States, published studies are not so much, 
probably reflecting a lower spread of necessary devices in these countries. ${ }^{[25,28-38]}$

Similarly, clinical and prognostic information of carotid RF-QAS is still limited in the international literature; however, in addition to traditional parameters, also, arterial stiffness (RF-QAS) values could be very useful in helping to understand arteriosclerosis, its severity, and progression. Arterial stiffness is an independent predictive marker of CV events. RF-QAS may help detect early patients with high-speed PWV relative to their age group, reflecting early atherosclerotic impregnation that occurs before the diagnosis of stiffness according to the value published in various pathological studies $(12 \mathrm{~m} / \mathrm{s})$. This technique is the most innovative and accurate method in the evaluation of the "state of health of blood vessels." Probably, accurate CV management at an early stage can provide a benefit to efficiently plan prevention and treatment. ${ }^{[16,39]} \mathrm{PWV}$ measurement is used to evaluate arterial stiffness in real time. This measure is intended to assess the degree of aging of the arterial system and its influence on the central pressure and pulse (clinical markers). RF-QAS measures carotid artery's wall stiffness and represents a truly study of the functional remodeling of the artery. Arterial wall stiffness is expressed as the PWV obtained from the brachial blood pressure and the accurate measurements of diameter and diameter variation. Local blood pressure at the site of the ultrasound measurement is also measured. Through dedicated algorithms, RF-QAS associates the parameters of relaxation of the walls of the vessels to the local blood pressure, thus determining the vascular stiffness. ${ }^{[16]}$ The importance of RF-QAS technology is based on the accurate resolution of the measurement. By analyzing RF, it is possible to reach greater independence and greater measurement accuracy as compared to all other available solutions. ${ }^{[7,40]}$

\section{Global Longitudinal Strain}

Conventional echocardiography allows us to obtain several parameters to describe the anatomy and function of cardiac structures. It allows quantification of left ventricular (LV) structure and function from the geometric measurements such as internal diameters, wall thickness, volumes, mass to global and regional systolic function (ejection fraction [EF], wall motion), and diastolic function (tissue Doppler imaging [TDI]). Cardiac-derived hemodynamics is detectable by Doppler flow and tissue Doppler analysis.

LV asymptomatic systolic dysfunction is an incidental finding during ambulatory visits, or it may be diagnosed during hospitalization. It has strong adverse prognostic implications. An appropriate stratification of these patients by echo to allow the identification of the causes of systolic (or diastolic impairment) should be mandatory for an efficient preventive strategy. ${ }^{[41,42]}$

Diastolic dysfunction is a common finding in patients at risk for developing heart failure with preserved EF. Sometimes, earlier systolic abnormalities coexist with diastolic impairment. Indeed, stage B heart failure (cardiac involvement without any previous or present sign/symptom) represents the vast majority of cases (about $70 \%$ of heart failure patients' burden). Actually, technological development has made available pocket size echocardiographic devices. These devices, with low cost and fundamental functions, are becoming useful partners of diagnostic routine clinical approach because of the obvious added potential of echocardiography over simple clinical examination. ${ }^{[43]}$

There is actually great interest in myocardial deformation imaging, actually estimated by speckle tracking echocardiography (STE).

STE is a newer technique, based on frame-by-frame tracking of small rectangular speckle patterns within the myocardial region of interest (ROI) on gray-scale echocardiographic images. The tracking algorithm creates a model of the chamber of interest after manual delineation of the endocardial border. Myocardial strain by speckle tracking is directly calculated from the variation of the segment length ("Lagrangian" strain). Thus, at a given time $t_{i}: S_{i}\left(L_{i}-L_{0}\right) / L_{0}$, where $S_{i}$ is strain at time $t_{i}, L_{i}$ is the segment length at $t_{i}$, and $L_{0}$ is the segment length at onset of QRS (end-diastolic length theoretical point of zero strain).

Strain rate is calculated as strain temporal derivative. Global strain and strain rate are calculated using the entire length of the tracked myocardium as baseline length. This approach is suitable for assessment of both long- and short-axis mechanics. The validity of myocardial mechanics measurements (strain and strain rate, both systolic and diastolic) with speckle tracking has been extensively validated by sonomicrometry in animals and with cardiac magnetic resonance (CMR) in humans. Global values of strain measurements, which are reproducible parameters of global systolic function, have been demonstrated to have superior prognostic properties over LVEF in major observational studies. In particular, speckle tracking is also suitable for right ventricular (RV) function assessment. ${ }^{[41,44-48]}$

The technique is semi-automated, with fewer possibilities of human errors. Inter-observer and especially intra-observer reproducibility of global strain is superior to $\mathrm{EF}$ as a measure of global LV systolic function; furthermore, it has strong prognostic implications after an acute myocardial infarction, superior to that of biplane LVEF. In addition, speckle tracking has a superior reproducibility compared with TDI for the assessment of regional and global ventricular mechanics in head-to-head studies. Finally, test-retest reproducibility of mitral annular velocities, an important component of diastolic function assessment, appeared to be better with speckle tracking as compared to TDI in a detailed comparative study.

STE has recently allowed a noninvasive, fast, and reproducible assessment of the subtle alterations already present in the earlier phase of many pathological contexts such as hypertension, diabetes mellitus, silent ischemia, and cardiomyopathies. It is therefore pivotal to promote a wider application of the 
Di Bello, et al:: Cardio-ankle vascular index, local artery carotid stiffness and global longitudinal strain: A new paradigm for cardiovascular prevention

technique to have stronger reference values and prognostic correlates to alterations found.

Recent introduction of three-dimensional (3D) echocardiography, allowing a direct, fast, and noninvasive correlation of cardiac structural and functional parameters with other imaging techniques such as CMR imaging, opens new frontiers for reclassification of patients already evaluated with common parameters (EF; LV mass).

The Research group of SIEC is able to provide a wide network of expert researchers and centers on the field of application of ultrasounds in preventive cardiology. In the last years, several studies related to heart-vessel interaction and clinical application of arterial stiffness, as well as speckle tracking technology, using different techniques, have been published in international journals by the SIEC Research Group.

\section{AImS}

The aim of our project is to deeply observe a large study Italian population (preclinical stage) collected of both Class A (asymptomatic individuals at risk for developing CV events) and Class B (individuals with no symptoms but evidence of cardiac and vascular involvement and at risk for developing $\mathrm{CV}$ events) to achieve more punctual strategies to prevent clinical events and validate a simple, readily available clinical and instrumental approach with most solid parameters to use in daily clinical practice.

We will evaluate prospectively a large series of both normal subjects and patients with CV risk factors, to obtain (major objectives):

1. Reference normal values of CAVI and carotid RF-QAS, stratified for age, and gender (still lacking for Caucasian populations)

2. Correlation between CAVI, carotid RF-QAS, left and right functional remodeling, and CV risk factors in different clinical settings

3. Respective clinical role of CAVI and RF-QAS in predicting early and late signs of atherosclerosis (i.e., quality IMT [Q-IMT], carotid and aortic plaques, aortic thoracic and abdominal aneurysms, mitral annular calcification, and aortic valve sclerosis)

4. Respective clinical and prognostic role of speckle tracking echocardiography (global longitudinal strain [GLS]) in predicting $\mathrm{CV}$ events. Reproducibility of strain parameters

5. Cost-effectiveness analysis and quality control.

Each one of these objectives will be translated into a scientific publication, addressing the clinical value of these techniques.

Every year, with a predetermined schedule, will include one or more projects into clinical publications with a wide subsequent diffusion.

There will also be evaluated (minor objectives) side studies in single specific cohorts of the overall population as well as reproducibility assays (intra- and inter-operator) and clinical applicability of the methods.

\section{Methods}

A number of certified SIEC laboratories (5-10) will participate to the multicentric survey. Normal subjects and patients with CV risk factors will be enrolled (after signing a written informed consent, previously shared with Local Ethical Committee) and evaluated by means of:

- Clinical, pharmacological, and familial history

- $\quad$ ECG findings

- $\quad$ Risk functions (SCORE, JNC-VII, Framingham, and Progetto Cuore)

- Metabolic screening (glucose, insulin, homeostatic model assessment index, total cholesterol, high-density lipoprotein cholesterol, triglycerides, low-density lipoprotein cholesterol, uric acid, creatinine, estimated glomerular filtration rate): de novo or recent (within 6 months)

- CAVI measures

- Echo vascular at carotid and abdominal level, from standardized to novel parameters, including PWV, augmentation index, beta index, wave intensity, arterial compliance

- $\quad$ Carotid ultrasound with RF-QAS and Q-IMT

- Conventional 2D echocardiography with measures of LV and RV structure, remodeling and systolic function, diastolic function; tissue Doppler velocities at mitral annular site, LV and RV GLS

- Integrated cardiovascular approach, including coupling

- Using the same ESAOTE machine.

\section{Inclusion criteria}

- $\quad$ Age $\geq 18$ years

- Absence of CV risk factors

- $\quad$ Presence of CV risk factors without clinical events (diabetes mellitus, hypertension, dyslipidemia) according to SCORE, Framingham, and National (Progetto Cuore) risk charts.

Patients with previous CV events (stroke, transient ischemic attack, acute coronary syndromes, heart failure, and stable angina) will be excluded from the study.

An electronic case report form will include all above-mentioned parameters, to share a common database.

Each center will be able to complete and modify data online and will be able to perform statistical analysis on its own population. Administrator will dispose of all the datasets and will organize development of specific objectives for each center.

This group of patients will be observed for 3 years, with a deep workup every year/6 months (telephonically interview with predetermined questionnaire):

a. The first scope is to detect in this population the incidence of early subclinical abnormalities of CV function, related to metabolic, genetic, and lifestyle parameters

b. Another objective of the project is the detection of 
Di Bello, et al:: Cardio-ankle vascular index, local artery carotid stiffness and global longitudinal strain: A new paradigm for cardiovascular prevention

unexpected $\mathrm{CV}$ event of the examined subject (major or minor $\mathrm{CV}$ events) during follow-up period

c. The determination, on the basis of Cox multivariable analysis and Kaplan-Meier curves, of the prognostic impact of the above-mentioned parameters.

Patients will be asked for major CV events, introduction of therapies, clinical status, and laboratory findings.

\section{Financial support and sponsorship}

Esaote contributed by loaning a MyLab Alpha machine for the acquisition and analysis of study data.

\section{Conflicts of interest}

There are no conflicts of interest.

\section{RefERENCES}

1. Piepoli MF, Hoes AW, Agewall S, Albus C, Brotons C, Catapano AL, et al. 2016 European Guidelines on cardiovascular disease prevention in clinical practice: The Sixth Joint Task Force of the European Society of Cardiology and Other Societies on Cardiovascular Disease Prevention in Clinical Practice (constituted by representatives of 10 societies and by invited experts) Developed with the special contribution of the European Association for Cardiovascular Prevention \& Rehabilitation (EACPR). Eur Heart J 2016;37:2315-81.

2. Mancia G, Fagard R, Narkiewicz K, Redon J, Zanchetti A, Böhm M, et al. $2013 \mathrm{ESH} / \mathrm{ESC}$ guidelines for the management of arterial hypertension: The Task Force for the Management of Arterial Hypertension of the European Society of Hypertension (ESH) and of the European Society of Cardiology (ESC). Eur Heart J 2013;34:2159-219.

3. Vlachopoulos C,Aznaouridis K, Stefanadis C.Prediction of cardiovascular events and all-cause mortality with arterial stiffness: A systematic review and meta-analysis. J Am Coll Cardiol 2010;55:1318-27.

4. Di Bello V, La Carrubba S, Antonini-Canterin F, Di Salvo G, Caso P, La Canna G, et al. Role of electrocardiography and echocardiography in prevention and predicting outcome of subjects at increased risk of heart failure. Eur J Prev Cardiol 2015;22:249-62.

5. Mohammed $\mathrm{M}$, Zito $\mathrm{C}$, Cusmà-Piccione $\mathrm{M}$, Di Bella $\mathrm{G}$, Antonini-Canterin F, Taha NM, et al. Arterial stiffness changes in patients with cardiovascular risk factors but normal carotid intima-media thickness. J Cardiovasc Med (Hagerstown) 2013;14:622-8.

6. Di Salvo G, Di Bello V, Salustri A, Antonini-Canterin F, La Carrubba S, Materazzo C, et al. Early left ventricular longitudinal systolic dysfunction and cardiovascular risk factors in 1,371 asymptomatic subjects with normal ejection fraction: A tissue Doppler study. Echocardiography 2011;28:268-75.

7. Di Bello V, Carerj S, Perticone F, Benedetto F, Palombo C, Talini E, et al. Carotid intima-media thickness in asymptomatic patients with arterial hypertension without clinical cardiovascular disease: Relation with left ventricular geometry and mass and coexisting risk factors. Angiology 2009;60:705-13.

8. Antonini-Canterin F, Di Bello V, Di Salvo G, La Carrubba S, Bellieni G, Benedetto F, et al. Relation of carotid intima-media thickness and aortic valve sclerosis (from the ISMIR study ["Ispessimento Medio Intimale e Rischio Cardiovascolare"] of the Italian Society of Cardiovascular Echography). Am J Cardiol 2009;103:1556-61.

9. Antonini-Canterin F, Carerj S, Di Bello V, Di Salvo G, La Carrubba S, Vriz O, et al. Arterial stiffness and ventricular stiffness: A couple of diseases or a coupling disease? A review from the cardiologist's point of view. Eur J Echocardiogr 2009;10:36-43.

10. Vriz O, Pellegrinet M, Zito C, di Bello V, Bettio M, Carerj S, et al. One-point carotid wave intensity predicts cardiac mortality in patients with congestive heart failure and reduced ejection fraction. Int $\mathrm{J}$ Cardiovasc Imaging 2015;31:1369-78.

11. Vriz O, Zito C, di Bello V, La Carrubba S, Driussi C, Carerj S, et al. Non-invasive one-point carotid wave intensity in a large group of healthy subjects: A ventricular-arterial coupling parameter. Heart Vessels 2016;31:360-9.

12. Cusmà-Piccione $\mathrm{M}$, Zito $\mathrm{C}$, Khandheria $\mathrm{BK}$, Pizzino $\mathrm{F}$, Di Bella G, Antonini-Canterin $\mathrm{F}$, et al. How arterial stiffness may affect coronary blood flow: A challenging pathophysiological link. J Cardiovasc Med (Hagerstown) 2014;15:797-802.

13. Zito C, Mohammed M, Todaro MC, Khandheria BK, Cusmà-Piccione M, Oreto $\mathrm{G}$, et al. Interplay between arterial stiffness and diastolic function: A marker of ventricular-vascular coupling. J Cardiovasc Med (Hagerstown) 2014;15:788-96

14. Vriz O, Bossone E, Bettio M, Pavan D, Carerj S, Antonini-Canterin F. Carotid artery stiffness and diastolic function in subjects without known cardiovascular disease. J Am Soc Echocardiogr 2011;24:915-21.

15. Carerj S, Penco M, La Carrubba S, Salustri A, Erlicher A, Pezzano A; Investigators of the DAVES Study. The DAVES (Disfunzione Asintomatica VEntricolare Sinistra) study by the Italian Society of Cardiovascular Echography: Rationale and design. J Cardiovasc Med (Hagerstown) 2006:7:457-63.

16. Castellon X, Bogdanova V. Screening for subclinical atherosclerosis by noninvasive methods in asymptomatic patients with risk factors. Clin Interv Aging 2013;8:573-80.

17. Van Bortel LM, Laurent S, Boutouyrie P, Chowienczyk P, Cruickshank JK, De Backer T, et al. Expert consensus document on the measurement of aortic stiffness in daily practice using carotid-femoral pulse wave velocity. J Hypertens 2012;30:445-8.

18. Ticulescu CR, Vriz O, Sparacino L, Popescu BA, Ginghina C, Nicolosi GL, et al. Incremental value of arterial stiffness over traditional risk factors in predicting subclinical cardiovascular remodeling in patients with moderate chronic renal failure. Angiology 2011;62:662-8.

19. Miyoshi T, Ito H. Assessment of arterial stiffness using the cardio-ankle vascular index. Pulse (Basel) 2016;4:11-23.

20. Mitchell GF, Hwang SJ, Vasan RS, Larson MG, Pencina MJ, Hamburg NM, et al. Arterial stiffness and cardiovascular events: The Framingham Heart Study. Circulation 2010;121:505-11.

21. Sutton-Tyrrell K, Najjar SS, Boudreau RM, Venkitachalam L, Kupelian V, Simonsick EM, et al. Elevated aortic pulse wave velocity, a marker of arterial stiffness, predicts cardiovascular events in well-functioning older adults. Circulation 2005;111:3384-90.

22. Ueki Y, Miura T, Minamisawa M, Abe N, Nishimura H, Hashizume N, et al. The usefulness of brachial-ankle pulse wave velocity in predicting long-term cardiovascular events in younger patients. Heart Vessels 2016. [Epub ahead of print].

23. Willum-Hansen T, Staessen JA, Torp-Pedersen C, Rasmussen S, Thijs L, Ibsen $\mathrm{H}$, et al. Prognostic value of aortic pulse wave velocity as index of arterial stiffness in the general population. Circulation 2006;113:664-70.

24. Mattace-Raso FU, van der Cammen TJ, Hofman A, van Popele NM, Bos ML, Schalekamp MA, et al. Arterial stiffness and risk of coronary heart disease and stroke: The Rotterdam Study. Circulation 2006; 113:657-63.

25. Wang H, Shirai K, Liu J, Lu N, Wang M, Zhao H, et al. Comparative study of cardio-ankle vascular index between Chinese and Japanese healthy subjects. Clin Exp Hypertens 2014;36:596-601.

26. Horinaka S, Yagi H, Ishimura K, Fukushima H, Shibata Y, Sugawara R, et al. Cardio-ankle vascular index (CAVI) correlates with aortic stiffness in the thoracic aorta using ECG-gated multi-detector row computed tomography. Atherosclerosis 2014;235:239-45.

27. Sun CK. Cardio-ankle vascular index (CAVI) as an indicator of arterial stiffness. Integr Blood Press Control 2013;6:27-38.

28. Park HE, Choi SY, Kim MK, Oh BH. Cardio-ankle vascular index reflects coronary atherosclerosis in patients with abnormal glucose metabolism: Assessment with 256 slice multi-detector computed tomography. J Cardiol 2012;60:372-6.

29. Yambe T, Yoshizawa M, Saijo Y, Yamaguchi T, Shibata M, Konno S, et al. Brachio-ankle pulse wave velocity and cardio-ankle vascular index (CAVI). Biomed Pharmacother 2004;58 Suppl 1:S95-8.

30. Kim B, Takada K, Oka S, Misaki T. Influence of blood pressure on cardio-ankle vascular index (CAVI) examined based on percentage change during general anesthesia. Hypertens Res 2011;34:779-83.

31. Suzuki J, Sakakibara R, Tateno F, Tsuyusaki Y, Kishi M, Ogata T, et al. 
Di Bello, et al.: Cardio-ankle vascular index, local artery carotid stiffness and global longitudinal strain: A new paradigm for cardiovascular prevention

Parkinson's disease and the cardio-ankle vascular stiffness index. Intern Med 2014;53:421-6.

32. Homma S, Kato K, Hayashi J, Yamamoto M. Negative associations between arterial stiffness parameter evaluated by cardio-ankle vascular index and serum low-density lipoprotein cholesterol concentration in early-stage atherosclerosis. Angiology 2015;66:143-9.

33. Nagayama D, Endo K, Ohira M, Yamaguchi T, Ban N, Kawana H, et al. Effects of body weight reduction on cardio-ankle vascular index (CAVI). Obes Res Clin Pract 2013;7:e139-45.

34. Yukutake T, Yamada M, Fukutani N, Nishiguchi S, Kayama H, Tanigawa T, et al. Arterial stiffness determined according to the cardio-ankle vascular index (CAVI) is associated with mild cognitive decline in community-dwelling elderly subjects. J Atheroscler Thromb 2014;21:49-55.

35. Hu H, Cui H, Han W, Ye L, Qiu W, Yang H, et al. A cutoff point for arterial stiffness using the cardio-ankle vascular index based on carotid arteriosclerosis. Hypertens Res 2013;36:334-41.

36. Kalaycioglu E, Gökdeniz T, Aykan AÇ, Gül I, Dursun I, Kiris G, et al. The relationship between dipper/nondipper pattern and cardioankle vascular index in hypertensive diabetic patients. Blood Press Monit 2013;18:188-94

37. Kim H, Kim HS, Yoon HJ, Park HS, Cho YK, Nam CW, et al. Association of cardio-ankle vascular index with diastolic heart function in hypertensive patients. Clin Exp Hypertens 2014;36:200-5.

38. Soska V, Dobsak P, Dusek L, Shirai K, Jarkovsky J, Novakova M, et al. Cardio-ankle vascular index in heterozygous familial hypercholesterolemia. J Atheroscler Thromb 2012;19:453-61.

39. Zhang Y, Zheng R, Liu J, Zhang $\mathrm{H}, \mathrm{Xu}$ J. Ultrasonic radio frequency analysis technique for noninvasive evaluation of carotid artery intima-media thickness and elasticity in patients with dyslipidemia. Nan Fang Yi Ke Da Xue Xue Bao 2014;34:345-8.

40. Palombo C, Kozakova M, Guraschi N, Bini G, Cesana F, Castoldi G, et al. Radiofrequency-based carotid wall tracking: A comparison between two different systems. J Hypertens 2012;30:1614-9.

41. Mor-Avi V, Lang RM, Badano LP, Belohlavek M, Cardim NM, Derumeaux G, et al. Current and evolving echocardiographic techniques for the quantitative evaluation of cardiac mechanics: ASE/EAE consensus statement on methodology and indications endorsed by the Japanese Society of Echocardiography. Eur J Echocardiogr 2011;12:167-205.

42. Marwick TH, Leano RL, Brown J, Sun JP, Hoffmann R, Lysyansky P, et al. Myocardial strain measurement with 2-dimensional speckle-tracking echocardiography: Definition of normal range. JACC Cardiovasc Imaging 2009;2:80-4.

43. Conte L, Fabiani I, Barletta V, Bianchi C, Maria C, Cucco C, et al. Early detection of left ventricular dysfunction in diabetes mellitus patients with normal ejection fraction, stratified by BMI: A preliminary speckle tracking echocardiography study. J Cardiovasc Echogr 2013;23:73-80.

44. Ballo P, Cameli M, Mondillo S, Giacomin E, Lisi M, Padeletti M, et al Impact of diabetes and hypertension on left ventricular longitudinal systolic function. Diabetes Res Clin Pract 2010;90:209-15.

45. Stanton T, Leano R, Marwick TH. Prediction of all-cause mortality from global longitudinal speckle strain: Comparison with ejection fraction and wall motion scoring. Circ Cardiovasc Imaging 2009;2:356-64.

46. Conte L, Fabiani I, Pugliese NR, Giannini C, La Carruba S, Angelillis M, et al. Left ventricular stiffness predicts outcome in patients with severe aortic stenosis undergoing transcatheter aortic valve implantation. Echocardiography 2017;34:6-13.

47. Fabiani I, Scatena C, Mazzanti CM, Conte L, Pugliese NR, Franceschi S, et al. Micro-RNA-21 (biomarker) and global longitudinal strain (functional marker) in detection of myocardial fibrotic burden in severe aortic valve stenosis: A pilot study. J Transl Med 2016;14:248.

48. Fabiani I, Conte L, Calogero E, Passiatore M, Pugliese NR, Santini V, et al. Review in translational cardiology: Micrornas and myocardial fibrosis in aortic valve stenosis, a deep insight on left ventricular remodeling. J Cardiovasc Echogr 2016;26:109. 